\title{
GMR
}

$\underline{\text { Review }}$

\section{Genetic tests for low- and middle-income countries: a literature review}

\author{
P.E. Maltese ${ }^{1}$, E. Poplavskaia ${ }^{2}$, I. Malyutkina ${ }^{2}$, F. Sirocco ${ }^{1}$, A. Bonizzato ${ }^{1}$, \\ N. Capodicasa ${ }^{3}$, S.Y. Nicoulina ${ }^{2}$, A. Salmina ${ }^{2}$, N. Aksutina ${ }^{2}$, M. Dundar ${ }^{4}$, \\ T. Beccari ${ }^{5}$, S. Cecchin ${ }^{1}$ and M. Bertelli ${ }^{1}$ \\ ${ }^{1}$ MAGI non-profit Human Medical Genetics Institute, Rovereto, Italy \\ ${ }^{2}$ Department of Internal Diseases N. 1, Krasnoyarsk State Medical University, \\ Krasnoyarsk, Russia \\ ${ }^{3}$ Faculty of Medicine, Catholic University “Our Lady of Good Counsel”, \\ Tirana, Albania \\ ${ }^{4}$ Department of Medical Genetics, Erciyes University Medical School, \\ Kayseri, Turkey \\ ${ }^{5}$ Department of Pharmaceutical Sciences, University of Perugia, Perugia, Italy \\ Corresponding author: P.E. Maltese \\ E-mail: paolo.maltese@assomagi.org
}

Genet. Mol. Res. 16 (1): gmr16019466

Received October 21, 2016

Accepted December 13, 2016

Published February 8, 2017

DOI http://dx.doi.org/10.4238/gmr16019466

Copyright (C) 2017 The Authors. This is an open-access article distributed under the terms of the Creative Commons Attribution ShareAlike (CC BY-SA) 4.0 License.

\begin{abstract}
The aim of this review is to describe a series of ten genetic diseases with Mendelian inheritance pattern in people of lowor middle-income countries, which can be easily identified with simple and affordable methods. Recent information shows that although genetic diseases account for more than $10 \%$ of infant mortality in such countries, testing, counseling, and treatment of genetic diseases is not a priority. The selection criteria for the genetic tests that are discussed in this review are: i) the frequency of the genetic disease in the general population, ii) the cost and ease of execution, and iii) the report of
\end{abstract}


validated methods in the literature for the diagnosis of these diseases. The goal is to promote diagnosis of genetic diseases at low-cost and with relative ease, thereby enabling appropriate treatments, reducing mortality, and preventing genetic diseases in high-risk families.

Key words: Low- and middle-income countries; Genetic tests; Mendelian genetic diseases; Literature review; Congenital anomalies; Infant mortality

\section{INTRODUCTION}

Birth defects or congenital anomalies, which often follow the Mendelian inheritance pattern, are a major cause of disabilities in developed and developing countries (Thong, 2014). Recent data shows that $10-30 \%$ of infant mortality in developing countries is due to genetic diseases (Kingsmore et al., 2012).

Diagnosis, counseling and treatment of genetic diseases is a significant burden on public health systems of many developed countries and also in low- or middle-income countries (LMICs), where health programs prioritize treatment of potentially lethal and acute diseases, such as infections or diseases arising from malnutrition.

According to the World Health Organization (WHO) experts, it is impossible to implement genetic programs, which are usually expensive, in very low-income countries as the level of financial opportunity, education, and technical development is too low to be sustained by the country economy (Kingsmore et al., 2012). Therefore, genetic screening programs are only feasible today in middle-income, transitioning, and developed countries.

In this review, we describe a series of ten genetic tests which can be used in LMICs. The choice of genetic diseases that need to be analyzed is a critical issue. Our first criterion was to focus on common diseases with Mendelian inheritance pattern (one-gene diseases) and multifactorial (complex, polygenic, and environment-interacting) diseases that can be diagnosed genetically (Table 1). Secondly, the results of the test should enable real improvement in the quality of life of the patients and their families (availability of effective sustainable treatments or responsible family planning). For example, a positive diagnosis of celiac disease can improve the quality of life through a simple change in diet and the identification of carriers of a recessive disease can help in family planning to reduce the number of affected children that are born. The Mendelian candidate diseases are cystic fibrosis (CF), spinal muscular atrophy (SMA), Duchenne/Becker muscular dystrophy (DMD/BMD), the fragile X syndrome (FXS), celiac disease (CD), adult type lactose intolerance, hereditary hemochromatosis (HH), familial Mediterranean fever (FMF), nonsyndromic deafness, and thrombophilia. Although diseases such as beta-thalassemia, phenylketonuria, sickle cell disease, hemophilia A, and Tay-Sachs disease are in the top ten Mendelian disorders in LMICs (Kingsmore et al., 2012), we did not include them in our study. This is because they can be diagnosed by ordinary laboratory methods without genetic tests.

To ensure sustainability (the number of samples to analyze will be large), genetic tests should be low-cost and easy to perform. Simple and affordable instruments such as a polymerase chain reaction (PCR) thermo cycler, an electrophoresis system, and a centrifuge should be sufficient for detection of the selected diseases and the entire start-up cost should not exceed 30,000 euros.

Genetics and Molecular Research 16 (1): gmr16019466 


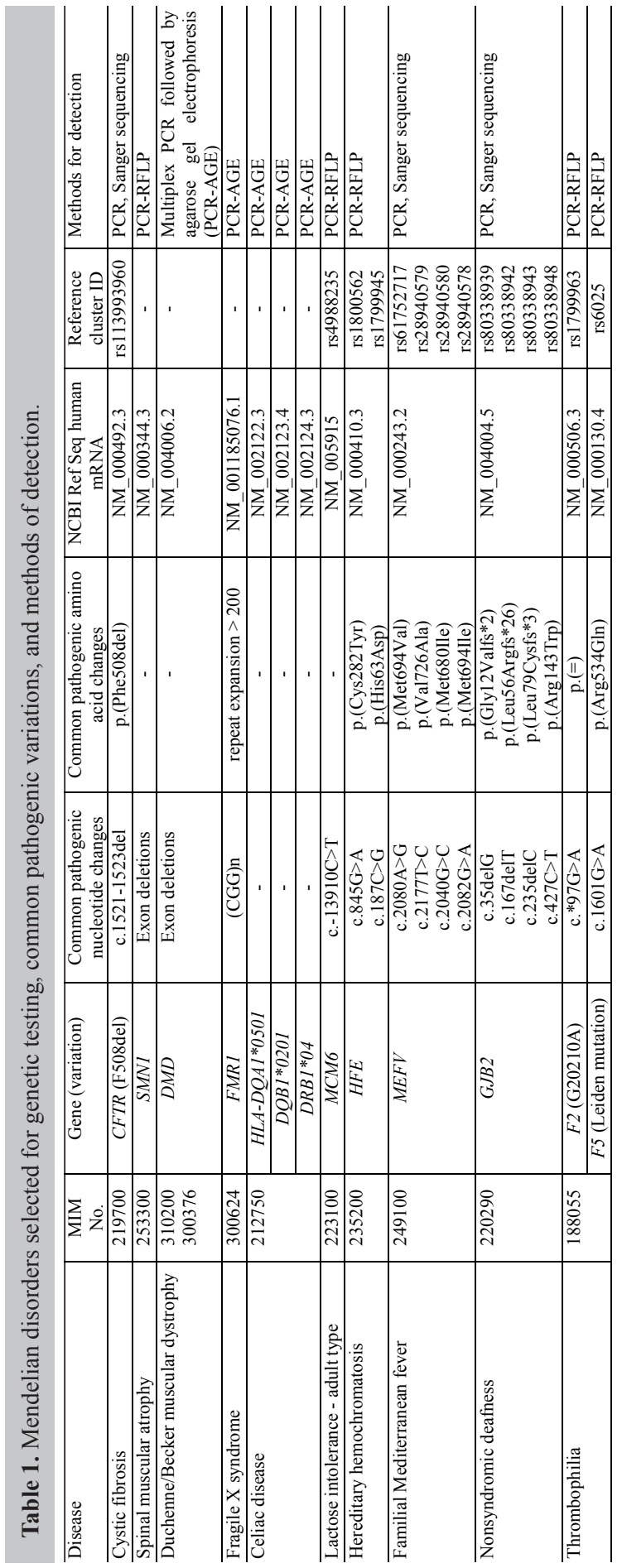

Genetics and Molecular Research 16 (1): gmr16019466 
All but three of the tests could be analyzed by PCR or PCR-restriction fragment length polymorphism (RFLP) (see list of primers in Table 2). Sanger sequencing was required for diagnosing cystic fibrosis, familial Mediterranean fever, and nonsyndromic deafness. Even though the diagnosis of these diseases is relatively expensive, we did not exclude them as nowadays the expenses for sequencing services is affordable.

Our approach is practically feasible since we have already initiated a pilot project, which has led to the establishment of two genetic screening laboratories in Tirana, Albania and in Krasnoyarsk, Russia.

\section{Table 2. List of primers.}

\begin{tabular}{|c|c|c|}
\hline Name of primer & Sequence & Disease \\
\hline E10-F & GCAGAGTACCTGAAACAGGA & Cystic fibrosis \\
\hline E10-R & CATTCACAGTAGCTTACCCA & Cystic fibrosis \\
\hline SMN_ex7_R111-F & AGACTATCAACTTAATTTCTGATCA & Spinal muscular atrophy \\
\hline SMN_ex7_X7-Dra-R & CCTTCCTTCTTTTTGATTTTGTTT & Spinal muscular atrophy \\
\hline SMN_ex8_541C960-F & GTAATAACCAAATGCAATGTGAA & Spinal muscular atrophy \\
\hline SMN_ex8_541C1120-R & CTACAACACCCTTCTCACAG & Spinal muscular atrophy \\
\hline DMD_exon_45-F & AAACATGGAACATCCTTGTGGGGAC & Duchenne/Becker muscular dystrophy \\
\hline DMD_exon_45-R & CATTCCTATTAGATCTGTCGCCCTAC & Duchenne/Becker muscular dystrophy \\
\hline DMD_exon_48-F & TTGAATACATTGGTTAAATCCCAACATG & Duchenne/Becker muscular dystrophy \\
\hline DMD_exon_48-R & CCTGAATAAAGTCTTCCTTACCACAC & Duchenne/Becker muscular dystrophy \\
\hline DMD_exon_19-F & GATGGCAAAAGTGTTGAGAAAAAGTC & Duchenne/Becker muscular dystrophy \\
\hline DMD_exon_19-R & TTCTACCACATCCCATTTTCTTCCA & Duchenne/Becker muscular dystrophy \\
\hline DMD_exon_17-F & GACTTTCGATGTTGAGATTACTTTCCC & Duchenne/Becker muscular dystrophy \\
\hline DMD_exon_17-R & AAGCTTGAGATGCTCTCACCTTTTCC & Duchenne/Becker muscular dystrophy \\
\hline DMD_exon_51-F & GAAATTGGCTCTTTAGCTTGTGTTTC & Duchenne/Becker muscular dystrophy \\
\hline DMD_exon_51-R & GGAGAGTAAAGTGATTGGTGGAAAATC & Duchenne/Becker muscular dystrophy \\
\hline DMD_exon_8-F & GGCCTCATTCTCATGTTCTAATTAG & Duchenne/Becker muscular dystrophy \\
\hline DMD_exon_8-R & GTCCTTTACACACTTTACCTGTTGAG & Duchenne/Becker muscular dystrophy \\
\hline DMD_exon_12-F & GATAGTGGGCTTTACTTACATCCTTC & Duchenne/Becker muscular dystrophy \\
\hline DMD_exon_12-R & GAAAGCACGCAACATAAGATACACCT & Duchenne/Becker muscular dystrophy \\
\hline DMD_exon_44-F & CTTGATCCATATGCTTTTACCTGCA & Duchenne/Becker muscular dystrophy \\
\hline DMD_exon_44-R & TCCATCACCCTTCAGAACCTGATCT & Duchenne/Becker muscular dystrophy \\
\hline DMD_exon_4-F & TTGTCGGTCTCTCTGCTGGTCAGTG & Duchenne/Becker muscular dystrophy \\
\hline DMD_exon_4-R & CAAAGCCCTCACTCAAACATGAAGC & Duchenne/Becker muscular dystrophy \\
\hline DMD_exon_46-F & GCTAGAAGAACAAAAGAATATCTTGTC & Duchenne/Becker muscular dystrophy \\
\hline DMD_exon_46-R & CTTGACTTGCTCAAGCTTTTCTTTTAG & Duchenne/Becker muscular dystrophy \\
\hline c-primer-F & GCTCAGCTCCGTTTCGGTTTCACTTCCGGT & Fragile $\mathrm{X}$ syndrome \\
\hline f-primer-R & AGCCCCGCACTTCCACCACCAGCTCCTCCA & Fragile $\mathrm{X}$ syndrome \\
\hline DQA1*0501-F & AGCAGTTCTACGTGGACCTGGGG & Celiac disease \\
\hline DQA1*0501-R & GGTAGAGTTGGAGCGTTTAATCAGA & Celiac disease \\
\hline DQB1*0201-F & CGCGTGCGTCTTGTGAGCAGAAG & Celiac disease \\
\hline DQB1*0201-R & GGCGGCAGGCAGCCCCAGCA & Celiac disease \\
\hline DRB1*04-F & GGTTAAACATGAGTGTCATTTCTTAAAC & Celiac disease \\
\hline DRB1*04-R & GTTGTGTCTGCAGTAGGTGTC & Celiac disease \\
\hline MCM6-F & GCTGGCAATACAGATAAGATAATGGA & Lactose intolerance \\
\hline MCM6-R & CTGCTTTGGTTGAAGCGAAGAT & Lactose intolerance \\
\hline $\mathrm{C} 282 \mathrm{Y}-\mathrm{F}$ & GGGTATTTCCTTCCTCCAACC & Hereditary hemochromatosis \\
\hline C282Y-R & CTCAGGCACTCCTCTCAACC & Hereditary hemochromatosis \\
\hline H63D-F & ACATGGTTAAGGCCTGTTGC & Hereditary hemochromatosis \\
\hline H63D-R & GCCACATCTGGCTTGAAATT & Hereditary hemochromatosis \\
\hline MEFV_ex10-F & ATTTTCTTTGCAGTTAATGTGATTC & Familial Mediterranean fever \\
\hline MEFV_ex10-R & GATACAAGGCCAGAAGCAGG & Familial Mediterranean fever \\
\hline GJB2_ex2_ampl-F & TGCTTACCCAGACTCAGAGAA & Nonsyndromic deafness \\
\hline GJB2_ex2_ampl-R & CGACTGAGCCTTGACAGCTGA & Nonsyndromic deafness \\
\hline GJB2_seq-F & AGTGGCCATGCACGTGGCCTA & Nonsyndromic deafness \\
\hline GJB2_seq-R & TGATCTCCTCGATGTCCTTAA & Nonsyndromic deafness \\
\hline FII_20210_G $>$ A_F & TCTAGAAACAGTTGCCTGGC & Thrombophilia \\
\hline FII_20210_G $>$ A_R & ATAGCACTGGGAGCATTGAAGC & Thrombophilia \\
\hline FV_F & TGCCCAGTGCTTAACAAGACCA & Thrombophilia \\
\hline FV_R & CTTGAAGGAAATGCCCCATTA & Thrombophilia \\
\hline
\end{tabular}

Genetics and Molecular Research 16 (1): gmr16019466 


\section{CF}

$\mathrm{CF}$ is an autosomal recessive disease and the most fatal genetic disorder (Rosenstein and Cutting, 1998). CF affects the exocrine function of the pancreas, the bile tract (biliary cirrhosis), the intestinal glands (meconium ileus), sweat glands, and bronchial glands (chronic bronchopulmonary infection with emphysema), and sometimes manifests as infertility. Symptoms of CF vary depending on the severity of the disease, eventually leading to death if the lung is involved. In most cases, CF is suspected on the basis of one or more typical clinical features such as the presence of a CF sibling or a positive result in a newborn screening test that detects the presence of elevated concentrations of chloride in sweat ( $>60 \mathrm{mM}$ ) (Rosenstein and Cutting, 1998).

CF occurs in 1:3200 people among Caucasians (Rosenstein and Cutting, 1998) and may occur with a lower frequency in other ethnic groups (1:15,000 in African Americans and 1:31,000 in Asian Americans). The only gene known to be associated with CF is the CF transmembrane conductance regulator gene (CFTR), which has more than 1500 identified mutations.

\section{Method suggested to LMICs}

The mostcommon CFTR variation is a deletion of threenucleotides c.1521_1523delCTT that results in the loss of a phenylalanine residue located at position 508 in the protein and is therefore referred as p.(Phe508del) (best known as F508del). The presence of this variant will produce an abnormal CFTR protein with impaired function. The consequent anomaly in chlorine excretion leads to secretion of dense, viscous mucous that obstructs major ducts causing multi-systemic defects.

The variant can be detected either by an amplification refractory mutation system (ARMS) (Ferrie et al., 1992) or by PCR amplification of exon 10 followed by direct sequencing (Ni et al., 2012). The ARMS methodology is simple, rapid, reliable, and inexpensive and can be easily afforded by LMICs. Additionally, PCR followed by direct sequencing could be a feasible approach; however, LMICs will need to avail the sequencing services at additional costs.

\section{Clinical utility of the diagnostic test}

The ultimate goal of $\mathrm{CF}$ treatment is to preserve lung function. Current therapies include use of antibiotics, mucolytics, broncholytics, and pancreatic enzymes (Rosenstein and Cutting, 1998). Home management methods such as airway clearance, medication, nutritional advice, and exercise increase life expectancy although the benefits largely depend on the conscientiousness of the patient with the daily home-based treatments. Genetic tests aimed at identifying carriers in the population, especially in countries where the frequency is high, can be important in family planning and in controlling the spread of the disease.

\section{SMA}

SMA, like CF, is an autosomal recessive disorder and the second fatal genetic disease. SMA involves progressive muscle weakness and symmetrical proximal paralysis resulting from loss and degeneration of anterior horn cells in the brain stem nuclei and spinal cord. The incidence of the disorder varies between 1:6000 and 1:10,000 and the frequency of carriers is approximately 1:50 (Monani and De Vivo, 2014).

Genetics and Molecular Research 16 (1): gmr16019466 
There are three types of SMA (I, II, and III) that depend on the copy number of survival motor neuron 2 (SMN2) gene, which ranges from zero to five in the population; the higher the number of SMN2, the greater is the quantity of functional SMN and the less severe is the disease. Sometimes an adult-onset type IV is also indicated (Monani and De Vivo, 2014). This classification has persisted for historical reasons. Today, scientists believe that the boundaries between the classes are less distinct and that patients show a spectrum of SMA severity from the onset in utero (truly severe) to the onset in middle or advanced age (very mild). Although clinical severities vary widely, all SMA patients have typical muscle weakness that affects the proximal muscles more than the distal ones, the lower limbs more than the upper limbs, and the intercostal and axial muscle groups more than the diaphragm (Monani and De Vivo, 2014). Earlier, clinicians used electromyography and biopsy features such as denervation of muscles for diagnosis; however, molecular testing for SMN1 now allows efficient and specific diagnosis.

To delay the disease progression and prolong patients' lives, basic nutritional and respiratory care and physiotherapy are required. Some new drugs are being tested, and others, such as valproic acid, are already known. Paralysis can be halted but not reversed (Baioni and Ambiel, 2010).

\section{Method suggested to LMICs}

The disease is caused by variations in SMN (Monani and De Vivo, 2014), which exists in two nearly identical copies, SMN1 in the telomeric region of chromosome 5 and SMN2 near the centromeric region of the same chromosome. Genetic variations reduce the levels of SMN, leading to degeneration of alpha motor neurons in the spinal cord (Monani and De Vivo, 2014). A normal individual (wild type) can possess both SMN1 and SMN2 or possess SMN1 alone that contributes to $90 \%$ of the SMN pool. In total, $95 \%$ of the patients have variations that arise from deletion or conversion of SMN1 to SMN2, leading to homozygous absence of exon 7 of SMN1 alone or in combination with exon 8. van der Steege et al. (1995) reported an easy, convenient, and rapid method in which PCR products, digested by restriction enzymes and visualized on a gel, revealed a specific pattern for SMA patients. PCR products of SMN exon 8 and the copied gene can be easily discriminated since the latter contains a recognition site (absent in $S M N$ exon 8) for the restriction enzyme DdeI. No difference in restriction site for any known enzyme exists for exon 7. Therefore, to detect the PCR product of exon 7 of the copied gene, van der Steege et al. (1995) developed an oligonucleotide adjacent to the variant site that carried a mismatch to create a restriction site for DraI.

\section{Clinical utility of the diagnostic test}

Because SMA is one of the most common lethal genetic disorders, direct carrier testing has been beneficial to many families (Monani and De Vivo, 2014). Genetic diagnostic testing for SMA is recommended since it does not require invasive muscle biopsy. Until an effective treatment is found, prenatal diagnosis is also recommended for prevention of new cases.

\section{DMD}

DMD is the most common clinical form of muscular dystrophy. It is an X-linked form caused by variations in the dystrophin gene, which is characterized by progressive muscle

Genetics and Molecular Research 16 (1): gmr16019466 
weakness and wasting due to absence of the protein dystrophin in muscles (Uwineza et al., 2014; van Ruiten et al., 2014). Increase in skeletal muscle damage and complete paralysis and death, usually because of respiratory and/or cardiac failure, occur in patients with DMD.

The disease affects approximately 1 in 3500 newborns. Diagnosis of DMD is based on careful analysis of clinical features and confirmed by further investigations such as muscle biopsy and/or genetic testing (De Palma et al., 2014). Elevated creatine kinase levels in blood can reflect muscle damage (van Ruiten et al., 2014). Nevertheless, DMD continues to be diagnosed too late.

BMD is a less severe variant of DMD caused by variations in the same gene.

\section{Method suggested to LMICs}

$\mathrm{DMD} / \mathrm{BMD}$ are caused by frame-shift deletions, duplications or nonsense variations in the dystrophin gene; deletions of one or more exons account for 55-65\% of cases of DMD and BMD (Forrest et al., 1988; Den Dunnen et al., 1989). A Multiplex PCR that amplify the most commonly deleted exons, is able to detect about $98 \%$ of deletions in patients with DMD/BMD (Chamberlain et al., 1988; Chamberlain et al., 1990; Beggs et al., 1991) by agarose gel electrophoresis.

\section{Clinical utility of the diagnostic test}

Treatment with glucocorticoids can slow disease progression and improve motor function in the short term. Active intervention is required in response to signs of respiratory or cardiac failure. In the last 20 years, scientists developed new therapeutic approaches for significantly improving the quality of life and life expectancy of patients with DMD (van Ruiten et al., 2014). Early diagnosis of DMD enables timely access to genetic counseling, standard care, and clinical trials.

\section{FXS}

The FXS is the most common inherited cause of intellectual disability and autism spectrum disorders worldwide. Expansion of a CGG repeat in the fragile $\mathrm{X}$ mental retardation 1 (FMR1) gene causes FXS (Tassone et al., 2008). The absence of FMR1 leads to decreased inhibition of expression of many synaptic proteins. FXS has a higher prevalence in males than in females (about 1 in 3600-4000 versus 1 in 4000-6000) (Cea-Del Rio and Huntsman, 2014). Physical features associated with FXS are prominent ears, long face, high-arched palate, macroorchidism, and hyperextensibility of finger joints. About $25 \%$ of females and $85 \%$ of males have cognitive impairment (IQ $<70$ ). They are also characterized by hyperactivity, attention deficiency, poor eye contact, shyness, self-talk, anxiety, mood instability, hyperarousal to sensory stimuli, and autism (Cea-Del Rio and Huntsman, 2014). A spectrum of medical problems, such as otitis media, seizures, and gastrointestinal problems, are commonly experienced by people with FXS.

Carrier screening is usually performed in the context of reproductive healthcare. Parents may be informed about reproductive options and family planning on the basis of the outcome. Newborn screening (NBS) for FXS was not recommended for inclusion in the panel of diseases requiring NBS in the 2006 American College of Medical Genetics report, partly because there was no medical advantage for early detection. However, recent reports from

Genetics and Molecular Research 16 (1): gmr16019466 
large screening studies that demonstrated the technical feasibility of widespread testing, the benefits of genetic counseling, and advances in treatment, may change this situation. In the case of FXS, identification of carriers not only enables counseling about the risk of having a child with FXS, but may also inform about personal health risks (Tassone, 2014).

\section{Method suggested to LMICs}

Tassone et al. (2008) reported a PCR-based screening method for FXS detection. Samples that yield PCR products with the standard primers, which is a single band for females (apparent homozygosity), or the absence of a band for males, undergo a second round of PCR screening with the c-primer and the CCG-chimeric primer that targets the CGG strand of the repeat expansion tract instead of the $\mathrm{f}$ primer.

\section{Clinical utility of the diagnostic test}

In the last few years we have entered a new age of targeted treatments, with a number of clinical trials for drugs such as minocycline, sertraline, mGluR5 antagonists, and gammaaminobutyric acid A and B receptor agonists, which show benefits and efficacy for patients with FXS, including young children. New targeted treatments will continue to develop and multiple family members with clinical involvement identified through neonatal diagnosis and familial cascade testing will also benefit from early treatment and long-term follow-up (Tassone, 2014). It is vital for pediatricians and other clinicians to be familiar with the medical problems related to FXS so that affected patients may receive proper diagnosis and treatment. Improved care may lead to better quality of life for these patients and their families.

\section{SUSCEPTIBILITY}

$\mathrm{CD}$, the most common food intolerance, is currently a major healthcare issue. $\mathrm{CD}$ is a chronic small-intestinal enteropathy triggered by gluten from wheat, barley, and rye (Green and Cellier, 2007; Tack et al., 2010). CD involves an autoimmune response in genetically susceptible individuals, leading to damage of the small intestinal mucosa, malabsorption of nutrients and malnutrition-related problems (Tack et al., 2010). Clinical manifestations of $\mathrm{CD}$ vary greatly according to age group. Infants and young children generally present with diarrhea, abdominal distension, and failure to thrive, although vomiting, irritability, anorexia, and constipation are also common. Adolescents and older children often show extra-intestinal manifestations, such as anemia, short stature and neurological symptoms (Green and Cellier, 2007). Recent estimates indicate that about $1 \%$ of the world population suffer from this disease. The disease is present not only in Europe and in countries populated by people of European ancestry, but also in the Asia, the Middle East, North Africa, and South America. $\mathrm{CD}$ is diagnosed on the basis of duodenal biopsy, serology, and response to gluten-free diet. However, about $10 \%$ of the cases are difficult to diagnose due to incongruence between clinical, serological, and histological findings (Green and Cellier, 2007).

\section{Method suggested to LMICs}

Although environmental factors that contribute towards pathogenesis of $\mathrm{CD}$ have been

Genetics and Molecular Research 16 (1): gmr16019466 
identified, the role of genetic risk factors is also well-known. Among the genes that are most involved, the best known is $H L A-D Q A 1$ and $H L A-D Q B 1$ (genes of the MHCII family that are highly polymorphic in the population). About $90 \%$ of individuals with CD carry the DQ2.5 $\left(H L A-D Q A 1^{*} 0501 / H L A-D Q B 1^{*} 02\right)$ haplotype and almost all others carry DQ8 (DQA1*03 / $D Q B 1 * 0302$ ) haplotype. At the same time, 30 and $10 \%$ of the general population harbor the DQ2.5 and DQ8 haplotypes, respectively.

Sacchetti et al. (1997) reported a rapid, accurate, high resolution, and cost-effective PCR-based method to analyze the genotypes of the HLA-DQA10501, DQB10201, and DRB104 alleles. The advantages of this method are rapid detection and non-requirement of radioactive material. Within 2 hours of sample DNA collection, researchers can visualize the three alleles that are most frequently associated with $\mathrm{CD}$ on the same gel. We suggest that this method could represent an additional tool in the $\mathrm{CD}$ diagnostic arsenal as it is suitable for epidemiological studies, family screening, and for diagnosis of cases where the histological and immunological patterns are ambiguous.

\section{Clinical utility of the diagnostic test}

Genetic testing such as HLA genotyping in the presence of symptoms could circumvent more invasive diagnostic methods that are based on biopsies and histopathological examination. Serological tests such as estimation of tissue transglutaminase levels and detection of autoantibodies against connective tissue endomysium exist. However, negative results in these tests does not guarantee that the subject will not develop the disease in the future (Tack et al., 2010). The only effective treatment for CD is to follow a gluten-free diet, which is sufficient in the overwhelming majority of patients. Clinical improvement is usually evident within a few weeks (Tack et al., 2010). Although the rate of diagnosis is increasing, CD remains undiagnosed in many affected people. The indications for screening include bloating, irritable bowel syndrome, thyroid disease, chronic unexplained diarrhea, chronic fatigue, and constipation. Many patients with CD seek healthcare for a variety of common symptoms. Therefore, frequent use of screening methods would assist in identifying new cases (Green and Cellier, 2007) and early diagnosis, which may reduce disease complications.

\section{ADULT TYPE LACTOSE INTOLERANCE}

Lactose intolerance is a gastrointestinal disorder with a wide range of symptoms. It is also known as lactase deficiency due to the absence of lactase-phlorizin hydrolase (LPH). Seventy-five per cent of the world population is intolerant to lactose; however, there is significant geographic variability, with a north-south frequency gradient going from $5 \%$ in northern European populations to $80-90 \%$ in some African and Asiatic populations (Sahi, 1994). A C/T polymorphism was identified in the MCM6 (2q21-22) gene, which is 13910 bp upstream of the gene encoding LPH ( $L C T)$ and is associated with the adult-onset form of lactose intolerance, also known as lactase non-persistence. The homozygous $\mathrm{C}$ variant, which is associated with lower gene transcription, correlates with the lactose-intolerance phenotype (Enattah et al., 2002). Depending on the cause of the disease, lactase deficiency is classified as primary, secondary or congenital (Heyman, 2006). The frequency of this disorder is above $50 \%$ in South America and Africa, while in certain Asian countries it can be close to $100 \%$. In general, $66 \%$ of the adult population of the world is lactase non-persistent. Secondary lactase

Genetics and Molecular Research 16 (1): gmr16019466 
deficiency is related to damage of the small intestine or other environmental causes. Congenital lactase deficiency (CLD) is a very rare and severe autosomal recessive gastrointestinal disorder of newborns due to mutations in $L C T$. Five common mutations are found in affected Finnish families and one of them - p.(Tyr1390*) - was the founder mutation, present in $90 \%$ of the disease alleles. Mutations in this gene have recently been found outside the Finnish population (Torniainen et al., 2009). CLD has an incidence of 1:60,000, is distributed globally, but is more frequent in Finland.

For newborn infants, ingestion of lactose causes symptoms so severe that breastfeeding becomes impossible. Severe osmotic diarrhea, followed by dehydration, acidosis, and weight loss usually afflict sufferers in the first days of life. Despite the symptoms, CLD infants are vigorous and hungry. Untreated disease leads to dehydration that usually requires hospitalization. When patients with various forms of lactase deficiency eliminate milk products from the diet, symptoms usually cease within short time. Calcium must be supplied by alternative non-dairy dietary sources or as a dietary supplement for individuals who avoid milk consumption.

Although rarely life-threatening, the symptoms of lactose intolerance can lead to significant discomfort, disrupted quality of life, leisure, and sporting activities, and loss of school/ workplace attendance. The disease, therefore, takes its toll on individuals, families, and society.

\section{Method suggested to LMICs}

Primary (late-onset) lactase deficiency is the most common cause of lactose intolerance and develops in various ages of childhood. It occurs due to certain common variations in a non-coding portion of $M C M 6$, which regulates expression of $L C T$.

Mulcare et al. (2004) reported a PCR-RFLP-based method for detection of lactose deficiency that includes introduction of a base change in the penultimate base of the MCM6_F primer to create a restriction site for HinfI in the T-containing allele, which can be subsequently used to digest the PCR product to produce two bands of 177 and $24 \mathrm{bp}$. If the $\mathrm{C}$ allele is present, the restriction site does not exist and therefore, the digestion results in a 201-bp product.

\section{Clinical utility of the diagnostic test}

Direct DNA-based diagnosis and carrier identification of CLD facilitate rapid intervention, elimination of milk products from the diet, and genetic counseling for this severe disease. Moreover, the genetic test detecting the c.-13910C $>$ T polymorphism is useful for differential diagnosis of lateonset congenital lactose intolerance in patients whose symptoms overlap with those of other lactase deficiency conditions such as damage to the intestinal mucosa from gastroenteritis (secondary deficit), chronic alcoholism, $\mathrm{CD}$, nutritional disorders, pharmacological therapies, and surgery.

\section{HH}

$\mathrm{HH}$ is an autosomal recessive disorder due to mutations in the hemochromatosis (HFE) gene. HFE encodes an atypical MHC class I molecule that regulates iron absorption, perturbations in which lead to iron accumulation in the body. Excess iron deposited in different organs results in serious illnesses, such as liver failure and cirrhosis, hepatocellular carcinoma, atherosclerosis, arthritis, fatigue, various endocrinopathies (including diabetes), heart problems (arrhythmia, cardiomyopathy, and loss of cardiac muscle function), hypermelanotic pigmentation of the skin, and immune deficiency (Vujić, 2014).

Genetics and Molecular Research 16 (1): gmr16019466 
The estimated carrier frequency of $\mathrm{HH}$ is $10 \%$ in people of northern European descent. In European patients with $\mathrm{HH}$, about $87 \%$ are either homozygous for the mutation causing p.(Cys282Tyr) or compound heterozygous for the mutations causing p.(Cys282Tyr) and p.(His63Asp) alterations in the protein primary sequence (Feder et al., 1996).

The HH symptom that most commonly impairs the quality of life is symmetrical polyarthritis involving small and large joints, and affects 30 to $60 \%$ of patients diagnosed with HH. Sclerosis, spurs, joint space narrowing, and chondrocalcinosis are evident in X-rays. The single most helpful screening test for $\mathrm{HH}$ is the determination of serum transferrin saturation. Serum ferritin is a highly sensitive marker for $\mathrm{HH}$ detection, albeit with low specificity. Therefore, a combination of ferritin and transferrin saturation testing is generally recommended for screening high-risk populations, including family screening, and in diagnostic testing in response to symptoms.

If untreated, $\mathrm{HH}$ is life-threatening. Treatment by phlebotomy to maintain serum ferritin concentrations at or below $50 \mathrm{ng} / \mathrm{mL}$ is simple and effective. Patients' quality of life can be improved by avoiding medicinal iron, mineral supplements, excess vitamin $\mathrm{C}$, and uncooked seafood.

Organ damage and iron loading can be avoided in patients with $\mathrm{HH}$ if prophylactic phlebotomy is carried out early in the disease. These findings emphasize the importance of early detection before onset of clinical symptoms.

\section{Method suggested to LMICs}

Oliveira et al. (2006) reported a PCR-RFLP-based method for HH detection. The p.(Cys282Tyr) variation creates a new cut site for RsaI. The digested PCR product produces two fragments of 145 and $296 \mathrm{bp}$ in wild-type alleles (Cys/Cys), three fragments $(29,116$, and $296 \mathrm{bp}$ ) in mutated alleles (Tyr/Tyr) while yields four fragments (29, 116, 145 and 296 $\mathrm{bp}$ ) in patients heterozygous (Cys/Tyr) for the variation. The p.(His63Asp) variation abolishes the $B c l$ recognition site, producing a product of $207 \mathrm{bp}$ while the wild-type sequence (His/ His) yields two fragments of 69 and $138 \mathrm{bp}$ and three fragments of 69, 138 and 207 when the variant is in heterozygous state.

\section{Clinical utility of the diagnostic test}

$\mathrm{HH}$ fits the accepted criteria for population screening by genetic testing: 1) it is relatively common in the targeted population, 2) it has a long asymptomatic period, allowing screening and treatment before occurrence of irreversible organ damage, 3) treatment is inexpensive and easily accessible, and 4) early intervention can prevent serious and life-threatening sequel, making population test a cost-effective plan to prevent illness and death. Iron loading and organ damage can be prevented in patients with hemochromatosis if prophylactic phlebotomy is carried out early in the disease, indicating the importance of early detection before clinical signs occur. In conclusion, targeted screening of populations at high risk of morbidity due to the $\mathrm{p}$.(Cys282Tyr) homozygosity (as seen in caucasian males) or p.(Cys282Tyr) and p.(His63Asp) compound heterozygosity by either biochemical analysis (for example, serum iron estimation) or genotype analysis is reasonable, cost-effective, and does not cause psychological harm, discrimination or stigma. To maximize costeffectiveness and minimize accumulation of ambiguous genetic information, it is recommended that genotypic screening probe only for the presence of the p.(Cys282Tyr) variant.

Genetics and Molecular Research 16 (1): gmr16019466 


\section{FMF}

FMF is an autosomal recessive disorder. FMF symptoms vary from recurrent attacks of fever, peritonitis, and pleuritis to inflammation of synovium, accompanied by pain and frequent amyloidosis with renal failure. FMF is more frequent in the south-eastern Mediterranean area; in non-Ashkenazi Jews, Armenians, Turks, and Arabs the average frequency is about 1-5 per 1000; it has a prevalence of up to 1:248 in the Libyan Jewish population and a carrier rate of 1:3-1:7 in North African Jews and Iraqi Jews (Almeida de Jesus and Goldbach-Mansky, 2013). The only genetic variations known to cause FMF occur in $M E F V$ at chromosomal locus 16p13. The typical features of FMF are frequent episodes of fever, which correlate with acute visceral pain, and large joint arthritis that persists for few days. Flares recur periodically from once a week to once a year with symptom-free intervals in between.

\section{Method suggested to LMICs}

Four common variants have been identified in exon 10 and less common mutations in exons 2, 3, 5, 9, and 10 of $M E F V$. Tchernitchko et al. (2005) reported direct sequencing of exon 10 as a diagnostic method.

\section{Clinical utility of the diagnostic test}

The diagnosis of FMF involves detection of variations in $M E F V$. Treatment of FMF included use of colchicine as a standard prophylaxis for inflammatory episodes and reactive amyloidosis in patients with FMF long before the genetic basis of FMF was understood. This treatment is now recommended for patients homozygous for the p.(Met694Val) variant or compound heterozygous for p.(Met694Val) and another disease-causing allele (Shohat and Halpern, 2011). In patients with poor response to colchicine, other therapies such as administration of azathioprine, thalidomide, and interferon alpha have been tested. Currently, IL-1 blockade with anakinra, canakinumab, and rilonacept has become the first choice for treatment of patients with poor response to colchicine although none of these drugs have yet been approved for use in the treatment of FMF (Gül, 2014).

Molecular genetic testing of $M E F V$ can be offered to family members of the patient, especially in cases that involve the p.(Met694Val) allele (Shohat and Halpern, 2011) as renal amyloidosis can be prevented by timely treatment.

\section{NONSYNDROMIC DEAFNESS}

Deafness is the most common sensory deficit in developed countries, affecting 1 every 500 newborns in Europe (Shearer and Smith, 2012), approximately 7 in every 1000 births in Nigeria and 5.5 in every 1000 births in South Africa (Bosch et al., 2014). Doctors can recognize some forms of genetic deafness by their associated syndromic features, but usually hearing failure is the only finding and is called nonsyndromic deafness (Duman and Tekin, 2012). Nonsyndromic hearing loss (NSHL) accounts for 70\% of hereditary deafness (Shearer and Smith, 2012).

The inheritance pattern of monogenic NSHL is autosomal recessive (ARNSHL) in approximately $80 \%$ of cases, autosomal dominant in about $20 \%$, X-linked in less than $1 \%$, and mitochondrial in $1 \%$ of cases. So far, researchers have discovered more than 700 different mutations in 1 of 65 genes causing nonsyndromic deafness.

Genetics and Molecular Research 16 (1): gmr16019466 
Gap junctions are abundantly present in the cochlea and enable diffusion of small molecules between interconnected cells. Connexins are transmembrane proteins that form hexameric hemichannels or connexons. Cochlear supporting cells preferentially express Cx26 and Cx30 isoforms of connexin. They work to maintain the $\mathrm{K}^{+}$homeostasis in the inner ear and are expressed in both epithelial and connective tissue gap junctions. GJB2, encoding Cx26, and GJB6, encoding Cx30, lie in close proximity to one another in the DFNB1 locus (Duman and Tekin, 2012).

\section{Method suggested to LMICs}

Reported mutations in GJB2 make this gene the most common cause of hearing loss in many populations (Duman and Tekin, 2012). However, this has not been seen in sub-Saharan Africa, except Ghana (Bosch et al., 2014).

Wu et al. (2003) reported the direct sequencing analysis of exon 2 of GJB2.

\section{Clinical utility of the diagnostic test}

Molecular genetic testing unequivocally diagnoses hereditary hearing loss and is useful to decide further patient management (Shearer and Smith, 2012). Identification of genetic markers of deafness may enable early detection and help in the choice of treatment.

\section{THROMBOPHILIA}

Thrombophilia is a major medical problem, and annually affects $0.1 \%$ of the population. The most common clinical manifestations are pulmonary embolism and venous thrombosis. Two single variants in the genes for coagulation factor $\mathrm{V}(F 5)$, the p.(Arg534Gln) best known as Factor V Leiden (FVL) variant and coagulation factor II $(F 2)$, the c.*97G $>$ A also known as prothrombin G20210A variant, are common in the general population and constitute major genetic risk factors for thrombosis (Dahlbäck, 2008).Compared to the general population, the risk of venous thrombosis is approximately three- to five-fold higher in individuals heterozygous for FVL and 80-fold in those homozygous for this mutation. The mutant protein is inactivated, slower than normal factor $\mathrm{V}$ and has longer half-life. The result is increased thrombin generation and mild hypercoagulability. Mutations in FVL are inherited as an autosomal dominant trait and is the most prevalent mutation in the Caucasian population (about 5\%), occurring in 3-7\% of individuals from the USA and Europe, but is extremely rare in Asian, African and indigenous Australian populations. FVL can be identified by functional APC-resistance tests or by DNA-based assays, which have about $100 \%$ sensitivity and specificity for the FVL mutation (Dahlbäck, 2008).

Factor II-related thrombophilia is the second-most common inherited contingency for thrombosis. The prothrombin G20210A is the historical name of a common single point variant in the 3'-UTR of the gene encoding factor II that increases prothrombin synthesis and concentration. Prothrombin-related thrombophilia is inherited in an autosomal dominant manner; heterozygosity for this variant (the mutation occurs near the polyadenylation site, increasing mRNA accumulation and protein synthesis) leads to increased risk of thrombosis. In the absence of other acquired or environmental predispositions, the relative risk is approximately two- to four-fold for heterozygotes (Martinelli et al., 2000). Recent studies have not included enough individuals homozygous for the " $\mathrm{A}$ " allele to evaluate the effect on recurrence risk.

Genetics and Molecular Research 16 (1): gmr16019466 
Similar to the FVL mutation, the prothrombin gene G20210A variant is more common among Caucasians. The frequency of occurrence of the heterozygote is approximately $2 \%$ in the general population, $3.0 \%$ in southern Europeans, and $1.7 \%$ in northern Europeans. It is very rare among persons of Asian and African descent (Safavi-Abbasi et al., 2008). Different studies have, however, shown that the association of prothrombin G20210A with FVL mutations not only increases the risk of developing venous thrombotic episodes, but also the risk of recurrences. In fact, heterozygotes for FVL and prothrombin G20210A (about 1 in 1000 Americans) had a 2.6-fold higher risk of recurrent thrombosis than carriers of FVL alone (Safavi-Abbasi et al., 2008) and a 20-fold higher risk than individuals with neither mutation, suggesting a multiplicative elevation in risk (Segal et al., 2009). This disorder can also be identified by DNA-based assays (Martinelli et al., 2000).

\section{Method suggested to LMICs}

FVL thrombophilia is due to the specific G-to-A substitution at nucleotide 1691 in F5 that results in amino-acid replacement (Arg506Gln) at one of three APC cleavage sites in the factor Va molecule (Safavi-Abbasi et al., 2008).

The $\mathrm{G}$ to A substitution at the 1691th bp in $F 5$ results in the loss of a recognition site for the restriction endonuclease MnlI. Voelkerding et al. (1996) utilized this to develop a PCRRFLP-based test for detecting the mutation.

Poort et al. (1996) reported using PCR followed by HindIII digestion to detect the prothrombin G20210A mutation in the 3'-UTR of the prothrombin gene using a mutagenic primer with a Hindlll site. The amplified products from the less-frequent allele (A) produce two fragments (322 and $23 \mathrm{bp}$ ) after enzyme digestion. The more-frequent allele (G) lacks the restriction site and therefore generates only a $345 \mathrm{bp}$ fragment upon digestion.

\section{Clinical utility of the diagnostic test}

Thrombophilia is a serious source of mortality and morbidity, and venous thromboembolism continues to present a challenge to clinicians. Individuals that harbor the FVL variant or the prothrombin G20210A variant are recommended to follow an oral anti-coagulation therapy (Safavi-Abbasi et al., 2008). In most cases venous thrombosis is first treated with a mixture of vitamin K antagonists and heparin (Dahlbäck, 2008). Because the incidence of these mutations seemed to significantly occur among people who died under 40 years of age, testing relatives of these patients could be useful for detection of at-risk individuals who would benefit from preventive anticoagulation therapy (Ranguelov et al., 2002).

\section{CONCLUSION}

Genetic counseling is urgently required in developing countries (Lee and Thong, 2013). In 2010, the WHO prioritized services for care and prevention of birth defects, particularly in LMICs. The development of genetic services in such countries is difficult for various reasons. Challenges include limited resources and low public awareness, ethical dilemmas such as religious and social issues and a shortage of genetic health professionals, especially genetic counselors (Lee and Thong, 2013).

In this study, we identified ten diseases of high incidence for which diagnosis in

Genetics and Molecular Research 16 (1): gmr16019466 
LMICs could improve the quality of life of patients or enable responsible family planning. Six of the ten diseases have autosomal recessive transmission (CF, SMA, adult type lactose intolerance, $\mathrm{HH}$, nonsyndromic deafness, and FMF), two have X-linked transmission (DMD and FXS), one has autosomal dominant transmission (thrombophilia), and another one has autosomal recessive/multifactorial transmission (CD). For some of these diseases, genetic analysis is focused only on exons or mutations cited in literature instead of the whole gene. This is limiting since genetic diseases must be tackled through correct diagnosis, safe and high quality patient care, and responsible procreation.

Many studies have demonstrated the importance of implementing genetic services for the care and prevention of congenital disorders in LMICs, but have received little attention. In a positive epidemiological transition, some middle- and a few low-income nations, such as Argentina, Bahrain, Brazil, Chile, China, Cuba, Egypt, India, Iran, Malaysia, Mexico, Oman, the Philippines, Saudi Arabia, Thailand, UAE, and Uruguay, have initiated and are developing medical genetic services for the care and prevention of congenital disorders. Neonatal screening for preventable childhood disorders and population screening to detect carriers of common recessive diseases are cost-effective when combined with genetic counseling and medical treatments and are beneficial to patients and their families in many countries (Kingsmore et al., 2012).

The main challenges for community genetics in LMICs are education of the population, creation of an infrastructure consisting of genetic professionals capable of offering appropriate counseling and follow-up, and appropriate consideration of core ethical principles. These requirements cannot be currently fulfilled in most developing countries and hence genetic tests are not enough to change lives of patients.

Here, we propose a model for enabling affordable and easy diagnosis of genetic diseases in LMICs. In our opinion, it is essential to build laboratories for diagnosis of genetic diseases and transfer the know-how directly to LMICs through the training of laboratory technicians and local doctors. These specialty laboratories should be established inside or in close proximity to research or medical centers (universities and hospitals), which can assist in setting up of the right collaborations between researchers or physicians and the diagnostic laboratories. Laboratory technicians should learn the techniques required to conduct simple and inexpensive tests for analyzing genetic diseases such as those described here and local doctors to recognize symptoms and record the family history of patients.

We have recently started two pilot test laboratories in Albania and Russia. The Albanian lab started its own activity last July by testing several patients for thrombophilia, celiac disease, and lactose intolerance. The Russian lab, which was set up in March 2016, is still undergoing the personnel training process.

We strongly believe that this model can be implemented by other LMICs and satisfy the urgent requirement of these countries for genetic screening-based diagnosis of congenital diseases. In addition, this venture could create attractive employment and research opportunities for biomedical students, scientists, and medical professionals and persuade them to return to their countries of origin.

\section{Conflicts of interest}

The authors declare no conflict of interest.

Genetics and Molecular Research 16 (1): gmr16019466 


\section{ACKNOWLEDGMENTS}

We are grateful to Helen Ampt for revising the manuscript.

\section{REFERENCES}

Almeida de Jesus A and Goldbach-Mansky R (2013). Monogenic autoinflammatory diseases: concept and clinical manifestations. Clin. Immunol. 147: 155-174. http://dx.doi.org/10.1016/j.clim.2013.03.016

Baioni MT and Ambiel CR (2010). Spinal muscular atrophy: diagnosis, treatment and future prospects. J. Pediatr. 86: 261-270.

Beggs AH, Hoffman EP, Snyder JR, Arahata K, et al. (1991). Exploring the molecular basis for variability among patients with Becker muscular dystrophy: dystrophin gene and protein studies. Am. J. Hum. Genet. 49: 54-67.

Bosch J, Lebeko K, Nziale JJ, Dandara C, et al. (2014). In search of genetic markers for nonsyndromic deafness in Africa: a study in Cameroonians and Black South Africans with the GJB6 and GJA1 candidate genes. OMICS 18: 481-485. http://dx.doi.org/10.1089/omi.2013.0166

Cea-Del Rio CA and Huntsman MM (2014). The contribution of inhibitory interneurons to circuit dysfunction in Fragile X Syndrome. Front. Cell. Neurosci. 8: 245.http://dx.doi.org/10.3389/fncel.2014.00245

Chamberlain JS, Gibbs RA, Ranier JE, Nguyen PN, et al. (1988). Deletion screening of the Duchenne muscular dystrophy locus via multiplex DNA amplification. Nucleic Acids Res. 16: 11141-11156. http://dx.doi.org/10.1093/ $\underline{\text { nar/16.23.11141 }}$

Chamberlain JS, Gibbs RA, Ranier JE and Caskey CT (1990). Multiplex PCR for the diagnosis of Duchenne muscular dystrophy. In: PCR Protocols: A Guide to Methods and Applications (MA Innis, DH Gelfand, JJ Sninsky, TJ White, eds). Academic Press, San Diego (USA). 272-281.

Dahlbäck B (2008). Advances in understanding pathogenic mechanisms of thrombophilic disorders. Blood 112: 19-27. http://dx.doi.org/10.1182/blood-2008-01-077909

De Palma C, Perrotta C, Pellegrino P, Clementi E, et al. (2014). Skeletal muscle homeostasis in duchenne muscular dystrophy: modulating autophagy as a promising therapeutic strategy. Front. Aging Neurosci. 6: 188.http://dx.doi. org/10.3389/fnagi.2014.00188

Den Dunnen JT, Grootscholten PM, Bakker E, Blonden LA, et al. (1989). Topography of the Duchenne muscular dystrophy (DMD) gene: FIGE and cDNA analysis of 194 cases reveals 115 deletions and 13 duplications. Am. J. Hum. Genet. 45: 835-847.

Duman D and Tekin M (2012). Autosomal recessive nonsyndromic deafness genes: a review. Front. Biosci. (Landmark Ed.) 17: 2213-2236. http://dx.doi.org/10.2741/4046

Enattah NS, Sahi T, Savilahti E, Terwilliger JD, et al. (2002). Identification of a variant associated with adult-type hypolactasia. Nat. Genet. 30: 233-237. http://dx.doi.org/10.1038/ng826

Feder JN, Gnirke A, Thomas W, Tsuchihashi Z, et al. (1996). A novel MHC class I-like gene is mutated in patients with hereditary haemochromatosis. Nat. Genet. 13: 399-408. http://dx.doi.org/10.1038/ng0896-399

Ferrie RM, Schwarz MJ, Robertson NH, Vaudin S, et al. (1992). Development, multiplexing, and application of ARMS tests for common mutations in the CFTR gene. Am. J. Hum. Genet. 51: 251-262.

Forrest SM, Cross GS, Flint T, Speer A, et al. (1988). Further studies of gene deletions that cause Duchenne and Becker muscular dystrophies. Genomics 2: 109-114.

Green PH and Cellier C (2007). Celiac disease. N. Engl. J. Med. 357: 1731-1743.http://dx.doi.org/10.1056/NEJMra071600

Gül A (2014). Treatment of familial Mediterranean fever: colchicine and beyond. Isr. Med. Assoc. J. 16: 281-284.

Heyman MB; Committee on Nutrition (2006). Lactose intolerance in infants, children, and adolescents. Pediatrics 118: 1279-1286. http://dx.doi.org/10.1542/peds.2006-1721

Kingsmore SF, Lantos JD, Dinwiddie DL, Miller NA, et al. (2012). Next-generation community genetics for low- and middle-income countries. Genome Med. 4: 25. http://dx.doi.org/10.1186/gm324

Lee JM and Thong MK (2013). Genetic counseling services and development of training programs in Malaysia. $J$. Genet. Couns. 22: 911-916. http://dx.doi.org/10.1007/s10897-013-9589-z

Martinelli I, Bucciarelli P, Margaglione M, De Stefano V, et al. (2000). The risk of venous thromboembolism in family members with mutations in the genes of factor V or prothrombin or both. Br. J. Haematol. 111: 1223-1229. http:// dx.doi.org/10.1046/j.1365-2141.2000.02502.x

Monani UR and De Vivo DC (2014). Neurodegeneration in spinal muscular atrophy: from disease phenotype and animal models to therapeutic strategies and beyond. Future Neurol. 9: 49-65.http://dx.doi.org/10.2217/fnl.13.58

Genetics and Molecular Research 16 (1): gmr16019466 
Mulcare CA, Weale ME, Jones AL, Connell B, et al. (2004). The T allele of a single-nucleotide polymorphism $13.9 \mathrm{~kb}$ upstream of the lactase gene (LCT) $(\mathrm{C}-13.9 \mathrm{kbT})$ does not predict or cause the lactase-persistence phenotype in Africans. Am. J. Hum. Genet. 74: 1102-1110. http://dx.doi.org/10.1086/421050

Ni WH, Jiang L, Fei QJ, Jin JY, et al. (2012). The CFTR polymorphisms poly-T, TG-repeats and M470V in Chinese males with congenital bilateral absence of the vas deferens. Asian J. Androl. 14: 687-690.http://dx.doi.org/10.1038/ aja. 2012.43

Oliveira TM, Souza FP, Jardim AC, Cordeiro JA, et al. (2006). HFE gene mutations in Brazilian thalassemic patients. Braz. J. Med. Biol. Res. 39: 1575-1580. http://dx.doi.org/10.1590/S0100-879X2006001200008

Poort SR, Rosendaal FR, Reitsma PH and Bertina RM (1996). A common genetic variation in the 3'-untranslated region of the prothrombin gene is associated with elevated plasma prothrombin levels and an increase in venous thrombosis. Blood 88: 3698-3703.

Ranguelov RD, Rosenthal N, Bromley C and Vasef MA (2002). Detection of factor V leiden and prothrombin gene mutations in patients who died with thrombotic events. Arch. Pathol. Lab. Med. 126: 1193-1196.

Rosenstein BJ and Cutting GR; Cystic Fibrosis Foundation Consensus Panel (1998). The diagnosis of cystic fibrosis: a consensus statement. J. Pediatr. 132: 589-595.http://dx.doi.org/10.1016/S0022-3476(98)70344-0

Sacchetti L, Sarrantonio C, Pastore L, Carlino V, et al. (1997). Rapid identification of HLA DQA1*0501, DQB1*0201 and DRB1*04 alleles in celiac disease by a PCR-based methodology. Clin. Chem. 43: 2204-2206.

Safavi-Abbasi S, Di Rocco F, Nakaji P, Feigl GC, et al. (2008). Thrombophilia Due to Factor V and Factor II Mutations and Formation of a Dural Arteriovenous Fistula: Case Report and Review of a Rare Entity. Skull Base 18: 135-143. http://dx.doi.org/10.1055/s-2007-1003926

Sahi T (1994). Hypolactasia and lactase persistence. Historical review and the terminology. Scand. J. Gastroenterol. Suppl. 202 (Suppl. 202): 1-6. http://dx.doi.org/10.3109/00365529409091739

Segal JB, Brotman DJ, Emadi A, Necochea AJ, et al. (2009). Outcomes of genetic testing in adults with a history of venous thromboembolism. Evid. Rep. Technol. Assess. (Full. Rep) 180: 1-162.

Shearer AE and Smith RJ (2012). Genetics: advances in genetic testing for deafness. Curr. Opin. Pediatr. 24: 679-686. http://dx.doi.org/10.1097/MOP.0b013e3283588f5e

Shohat M and Halpern GJ (2011). Familial Mediterranean fever--a review. Genet. Med. 13: 487-498. http://dx.doi. org/10.1097/GIM.0b013e3182060456

Tack GJ, Verbeek WH, Schreurs MW and Mulder CJ (2010). The spectrum of celiac disease: epidemiology, clinical aspects and treatment. Nat. Rev. Gastroenterol. Hepatol. 7: 204-213. http://dx.doi.org/10.1038/nrgastro.2010.23

Tassone F (2014). Newborn screening for fragile X syndrome. JAMA Neurol. 71: 355-359. http://dx.doi.org/10.1001/ jamaneurol.2013.4808

Tassone F, Pan R, Amiri K, Taylor AK, et al. (2008). A rapid polymerase chain reaction-based screening method for identification of all expanded alleles of the fragile X (FMR1) gene in newborn and high-risk populations. J. Mol. Diagn. 10: 43-49. http://dx.doi.org/10.2353/jmoldx.2008.070073

Tchernitchko D, Moutereau S, Legendre M, Delahaye A, et al. (2005). MEFV analysis is of particularly weak diagnostic value for recurrent fevers in Western European Caucasian patients. Arthritis Rheum. 52: 3603-3605. http://dx.doi. org/10.1002/art.21408

Thong MK (2014). Birth defects registries in the genomics era: challenges and opportunities for developing countries. Front Pediatr. 2: 60.http://dx.doi.org/10.3389/fped.2014.00060

Torniainen S, Savilahti E and Järvelä I (2009). Congenital lactase deficiency--a more common disease than previously thought?. Duodecim 125: 766-770.

Uwineza A, Hitayezu J, Murorunkwere S, Ndinkabandi J, et al. (2014). Genetic diagnosis of Duchenne and Becker muscular dystrophy using multiplex ligation-dependent probe amplification in Rwandan patients. J. Trop. Pediatr. 60: 112-117.http://dx.doi.org/10.1093/tropej/fmt090

van der Steege G, Grootscholten PM, van der Vlies P, Draaijers TG, et al. (1995). PCR-based DNA test to confirm clinical diagnosis of autosomal recessive spinal muscular atrophy. Lancet 345: 985-986. http://dx.doi.org/10.1016/S0140$\underline{6736(95) 90732-7}$

van Ruiten HJ, Straub V, Bushby K and Guglieri M (2014). Improving recognition of Duchenne muscular dystrophy: a retrospective case note review. Arch. Dis. Child. 99: 1074-1077. http://dx.doi.org/10.1136/archdischild-2014-306366

Voelkerding KV, Wu L, Williams EC, Hoffman SM, et al. (1996). Factor V R506Q gene mutation analysis by PCR-RFLP: optimization, comparison with functional testing for resistance to activated protein $\mathrm{C}$, and establishment of cell line controls. Am. J. Clin. Pathol. 106: 100-106. http://dx.doi.org/10.1093/ajcp/106.1.100

Vujić M (2014). Molecular basis of HFE-hemochromatosis. Front. Pharmacol. 5: 42.

Wu BL, Kenna M, Lip V, Irons M, et al. (2003). Use of a multiplex PCR/sequencing strategy to detect both connexin 30 (GJB6) $342 \mathrm{~kb}$ deletion and connexin 26 (GJB2) mutations in cases of childhood deafness. Am. J. Med. Genet. A. 121A: 102-108.http://dx.doi.org/10.1002/ajmg.a.20210

Genetics and Molecular Research 16 (1): gmr16019466 